\title{
Computational Analysis of Surface-Tension-Driven Coating-Defect Flow
}

\author{
Richard H. J . Blunk \\ G M R \&D Center, G eneral M otors Corp., W arren, MI 48090 \\ $J$ ames 0 . Wilkes \\ Dept. of Chemical Engineering, U niversity of M ichigan, A nn A rbor, MI 48109
}

\begin{abstract}
Bondline readout ( $B L R O)$ is a coating defect frequently exhibited on adhesivelybonded, polymeric automotive body panels painted with high-glamor/flow clearcoats. BLRO or telegraphing results from Marangoni-type, surface-tension-driven flows. An efficient numerical code was developed to predict temperature- and concentrationinduced BLRO flows to use it as an effective tool for screening and developing potential clearcoat systems. Two numerical codes were developed: a 1-D code based on the lubrication approximation; a 2-D code based on the SIMPLER (Semiimplicit method for pressure-linked equations revised) algorithm. The 1-D code produced BL RO profiles in agreement with those predicted from the 2-D code and those measured from BLRO flow experiments, thus confirming a proposed BLRO mechanism. Moreover, the 1-D code was vastly more time-efficient than the 2-D code due to a severe time-step stability limitation found with the latter.
\end{abstract}

\section{Introduction}

With the increased use of high-solids (and high-flow) clearcoats (B auer and Briggs, 1984) and customer-driven desired increase in coating reflectivity and smoothness, a large number of coating defects caused by Marangoni-type or surface-tension-driven flows (Pierce and Schoff, 1988) have become increasingly more prevalent in the automotive industry, especially on horizontal, polymeric composites sheet molding compound (SMC) body panels. One such defect, which is the topic of this article, is known as bondline readout (BLRO), or more generally as telegraphing, revealing structural features through the coated part after the coating has cured. $B L R O$ is caused by temperature and hence surface-tension gradients generated on the SM C surface during convection heating/curing of the paint. An adhesive bond, that is, the structural feature, acts as a heat sink and generates cooler temperatures on the SM C surface directly over the bond than away from it. Since surface tension increases with decreasing temperature, coating material is drawn from the non-bond regions, of low surface tension, to the bond regions, of high surface tension, resulting in thicker films over the bonds. The

\footnotetext{
Correspondence concerning this article should be addressed to R. H. J. Blunk.
}

end result is an undesirable outline or imprint in the clearcoat surface of the underlying adhesive bond, as shown in Figure 1. The bondline is, in effect, readout through the SM C panel and, subsequently, in the paint.

To eliminate BLRO, automotive manufacturers are forced to switch from polymeric to metallic body panels when using high-glamour/flow coatings, or from high-flow/high-glamour to low-flow/low-glamour coating systems when using polymeric body panels. Metal panels of high thermal diffusivity rarely produce significant temperature gradients, and lowflow coatings are not as susceptible to surface-tension-gradient forces. U nfortunately, in most cases, these changes result in weight and cost penalties or inferior vehicle finishes.

Leveling of thin liquid films on a horizontal surface has been studied for over forty years. O ne of the earliest theoretical analyses of surface leveling involved O rchard's linear theory, which investigated the decay of small-amplitude disturbances on the surface of $\mathrm{N}$ ewtonian liquids of uniform surface tension (O rchard, 1962). D egani and Gutfinger (1972) were first to model leveling of finite-amplitude disturbances on $\mathrm{N}$ ewtonian films by conducting a nonlinear analysis, using the finite-difference approximation scheme together with marker 


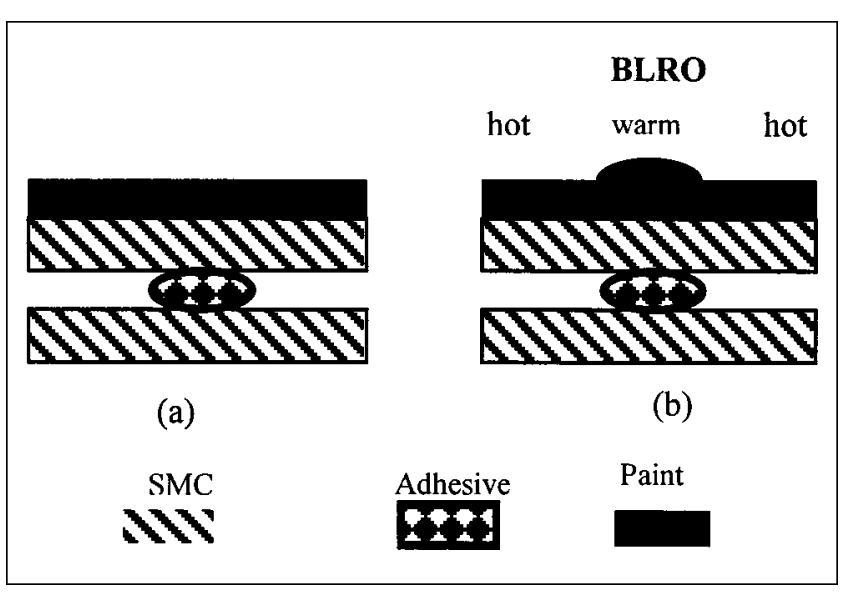

Figure 1. (a) Initial uniform clearcoat thickness before heating; (b) final nonuniform clearcoat thickness (BLRO) after heating (cross-sectional view).

particles to track the free surface. The surface tension, however, was incorrectly treated as a body force, not a surface force. A nonlinear finite-element analysis of leveling of $\mathrm{New}$ tonian films, under the influence of gravity and surface tension, was later conducted by K heshgi and Scriven (1983). The pressure was removed successfully from the momentum equations using a penalty formulation that enabled explicit time marching. Biermann (1968), using the linear theory of Orchard, was first to study the effects of elasticity on leveling of sinusoidal disturbances. Elasticity was found to retard leveling. Keunings and Bousfield (1987) also investigated viscoelastic effects using a nonlinear finite-element analysis to model leveling of a Oldroyd-B fluid. A gain, elasticity was found to decrease the leveling rate. Ellwood (1991) conducted a numerical analysis, using the finite-element method, to predict leveling of non-N ewtonian liquids and to investigate elasticity, inertia, and substrate geometry effects. R ecently, J oos (1996) studied leveling of viscoelastic films of long wavelengths and with stratified viscosity layers in the presence of insoluble surface-active materials. A change of the relative thickness of the different viscosity layers was found to affect leveling and the deformation of the individual layers.

The effects on leveling of concentration-induced surfacetension gradients, which are generated by solvent evaporation and are important in this BLRO study, have also been investigated. O verdiep (1986) demonstrated experimentally that Orchard's linearized theory for leveling was invalid for solvent-based alkyd paints, because it did not account for surface-tension-gradient effects, which were found to increase the leveling rate of Newtonian liquids. Moreover, these effects caused the surface disturbances to reappear after the paint film had become flat. The crests of the waves were formed where the valleys of the original surface waves were located and vice versa. The surface-tension gradients resulted from solvent concentration variations along the paint surface. These variations in turn resulted from solvent evaporating from a film of nonuniform thickness, similar to those formed in the "tears of strong wine" experiment (M arangoni, 1872).
A n integro-differential equation was developed, based on a modification of Orchard's theory, and solved numerically to predict these leveling effects. Eley, W eidner, and Schwartz (1996) extended Overdiep's work to include shear-thinning behavior and surface-active ingredients. Leveling was investigated using two models: one based on the lubrication approximation and the other based on the linearized theory of small-amplitude undulations. A gain, surface-tension gradients increased the rate of leveling. The surface-active substances retarded leveling by decreasing the surface-tension gradients.

The objective of this study is to develop an efficient numerical code for predicting deleveling (BLRO flow) - the opposite of leveling - where an initially, smooth, continuous, wet film of uniform thickness is subjected to temperature- and concentration-induced surface-tension gradients to produce surface nonuniformities (BLRO). Nevertheless, the leveling analyses described above are important in this study because $B L R O$ results from a combination of leveling and $M$ arangoni (surface-tension-gradient) flows. The code can then be used to obtain better insight into the BLRO mechanism by providing quantitative information regarding the relative effects of surface tension, gravity, and viscosity on BLRO, the velocity and pressure distributions, and the magnitude of temperature-induced vs. concentration-induced surface-tension gradients. The ultimate objective is to use the code as an efficient tool for screening and developing potential clearcoat systems, eliminating costly and time-consuming experimental work that is currently being done, so that polymeric composite vehicles can eventually be painted with high-glamour clearcoats without exhibiting BLRO.

T wo numerical codes are developed. O ne code, a 2-D code, solves the full 2-D Navier-Stokes equations without the inertia terms. The other code, a 1-D code, solves a nonlinear, fourth-order partial differential R eynolds equation based on the lubrication approximation. The codes predict the effects of temperature and concentration on film thickness and are developed specifically to handle simple coating materials and conditions, namely, non-reacting, single-solvent, single-resin films heated via conduction. Profile results from the two codes are compared, and results from the more efficient 1-D code are then compared with those obtained experimentally (Blunk, 1996). The codes can then be modified in future work to handle more complex automotive clearcoats and heating processes, namely, reacting, multiple-solvent, multiple-resin films heated via convection.

\section{Proposed BLRO Mechanism}

The BLRO film defect is shown in Figure 2. O nly half of the defect is illustrated due to symmetry. Four forces are involved in BLRO flow: (1) surface-tension gradient (driving force); (2) capillary pressure (leveling force); (3) viscosity (resisting force); and (4) gravity (leveling force). D uring the convection bake cycle, temperature variations exist across the film surfaces of adhesively-bonded, sheet molding compound (SMC) assemblies, with the cooler temperatures residing directly above the bond area, which acts as heat sink. M aterial is then drawn towards the cooler, bond area of high surface tension from the warmer, non-bond areas of low surface tension. 


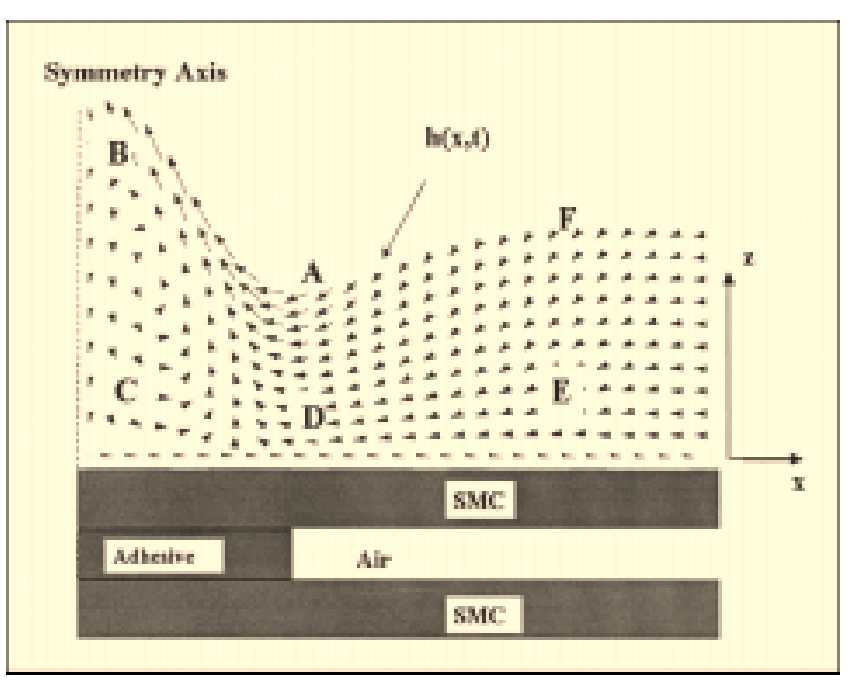

Figure 2. BLRO coating defect.

Only half of defect is shown due to symmetry.

The degree of BLRO depends on competing flowssurface-tension-gradient $M$ arangoni flow increases $B L R O$, while pressure-gradient flow decreases BLRO. The former results from temperature and/or concentration variations along the surface of a film. The latter results from nonuniform film thicknesses caused by $M$ arangoni flow. The pressures in the concave regions of the film ( $B$ and $F$ ) exceed that in the convex regions (A) due to capillary pressure effects. Pressure differences also exist at similar locations along the bottom of the film due to gravity or hydrostatic pressure effects with pressures at $C$ and $E$ exceeding that at $D$. Surfacetension gradients cause flow from $A$ to $B$, resulting in $B L R O$, while gravity and surface tension, together with surface curvature, cause pressure-gradient flow from $C$ and $E$ to $D$, towards the thin convex regions, resulting in leveling. The degree of BLRO also depends on time.

A ssuming the thin liquid film is Newtonian and the no-slip boundary condition is valid at the SM C surface, the velocity profiles through the film thickness for combined surface-tension-gradient and pressure-gradient flows are given by

$$
\mathrm{u}=\frac{\partial \sigma}{\partial \mathrm{x}} \frac{\mathrm{z}}{\mu}-\frac{\partial \mathrm{p}}{\partial \mathrm{x}} \frac{\mathrm{z}}{\mu}\left(\mathrm{h}-\frac{\mathrm{z}}{2}\right)
$$

where $u$ is the $x$-component of velocity and $\mu$ is the Newtonian viscosity (F ink-J ensen, 1962).

A mass balance shows that the time rate of change in film thickness $h$ is

$$
\frac{\partial \mathrm{h}}{\partial \mathrm{t}}=-\frac{\partial \mathrm{J}}{\partial \mathrm{x}}
$$

in which the flux $\mathrm{J}$ is obtained by integration of Eq. 1 over the film thickness ( $z=0$ to $h$ ):

$$
J=\frac{\partial \sigma}{\partial x} \frac{\mathrm{h}^{2}}{2 \mu}-\frac{\partial \mathrm{p}}{\partial \mathrm{x}} \frac{\mathrm{h}^{3}}{3 \mu} .
$$

By substituting Eq. 3 into Eq. 2, the time evolution of film thickness becomes

$$
\frac{\partial \mathrm{h}}{\partial \mathrm{t}}=-\frac{\partial^{2} \sigma}{\partial \mathrm{x}^{2}} \frac{\mathrm{h}^{2}}{2 \mu}-\frac{\partial \sigma}{\partial \mathrm{x}} \frac{\partial \mathrm{h}}{\partial \mathrm{x}} \frac{\mathrm{h}}{\mu}+\frac{\partial^{2} \mathrm{p}}{\partial \mathrm{x}^{2}} \frac{\mathrm{h}^{3}}{3 \mu}+\frac{\partial \mathrm{p}}{\partial \mathrm{x}} \frac{\partial \mathrm{h}}{\partial \mathrm{x}} \frac{\mathrm{h}^{2}}{\mu} .
$$

Since the film is initially of uniform thickness and pressure, Eq. 4 indicates that BLRO or a nonuniform film thickness can only be generated when the surface-tension gradient is not constant (that is, finite $\partial^{2} \sigma / \partial \mathrm{x}^{2}$ ), which occurs if the temperature gradient varies with position.

Temperature and concentration variations affect viscosity and surface tension, which in turn affect BLRO flow. Surface-tension effects are most evident with the occurrence of three modes of BLRO flow-formation, flow-out, and reformation. The magnitude of the shear-stress-driving force (Levich-A ris boundary condition) is given by

$$
\begin{aligned}
\tau=\nabla \sigma \cdot \overrightarrow{\mathrm{t}} \cong \frac{\partial \sigma}{\partial \mathrm{x}} & +\frac{\partial \mathrm{h}}{\partial \mathrm{x}} \frac{\partial \sigma}{\partial \mathrm{z}} \\
& \cong\left[\left(\frac{\overline{\partial \sigma}}{\partial \mathrm{T}}\right)\left(\frac{\partial \mathrm{T}}{\partial \mathrm{x}}\right)\right]+\left[\left(\frac{?}{\partial \sigma}\right)\left(\frac{\partial \overline{\mathrm{C}}}{\partial \mathrm{x}}\right)\right]
\end{aligned}
$$

where $\mathrm{h}$ is the film thickness, $\mathrm{C}$ is the solvent concentration, $T$ is the temperature, $\vec{t}$ is the unit vector tangent to the free surface, and $x$ and $z$ are coordinate directions. The surfacetension gradient is composed of two terms-the temperature-induced term (T-term) and the concentration-induced term (C-term). If the sum of the two terms is negative (positive), the shear stress acts in the negative (positive) $x$-direction. In Eq. 5, minus and plus signs are positioned directly over the partial derivatives. These signs are valid under all conditions used in this study. The signs for the temperature and concentration gradients are based on film conditions on the righthand side of the symmetrical profile, as presented in Figure 2. In the high-flow regions of interest, the temperatures near the symmetry line are always lower than that at larger $\mathrm{x}$-values, that is, $\partial \mathrm{T} / \partial \mathrm{x}$ is always positive. The sign of $\partial \sigma / \partial \mathrm{T}$ is always negative-surface tension decreases with increasing temperature. Thus, the T-term is always negative in this work and results in material flow towards the symmetry line (that is, BLRO formation and reformation). Since the solvent concentration is always greater under the BLRO hump compared to that in thin regions, that is, $\partial \mathrm{C} / \partial \mathrm{x}$ is always negative, due to solvent evaporation from a nonuniform thickness film, the sign of the C-term depends on the sign of its first partial derivative (unknown and delineated by a query "?'). If the solvent surface tension is less than that of the polymer resin, as is usually the case, then $\partial \sigma / \partial \mathrm{C}$ is negative and the $\mathrm{C}$-term is positive. A lso, if the magnitude of the $\mathrm{C}$ term is greater than that of the T-term, material flows away from the symmetry line (flow-out or leveling). $\mathrm{O} n$ the other hand, if $\partial \sigma / \partial \mathrm{C}$ is positive, the negative $\mathrm{C}$-term augments the negative $\mathrm{T}$-term and BLRO formation is enhanced. These temperature and concentration effects are demonstrated below. 


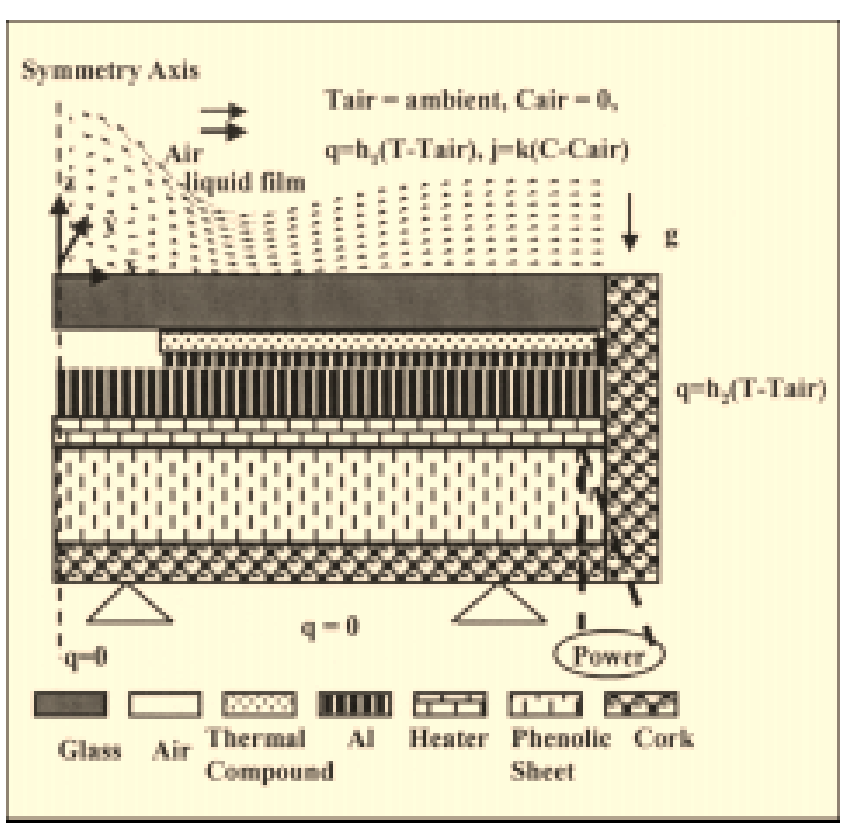

Figure 3. BLRO flow problem.

Cross-sectional view; not drawn to scale. Boundary conditions and conduction-heater materials are shown.

\section{BLRO Flow Problem}

The BLRO flow configuration, coordinates, and boundary conditions are presented in Figure 3, in which only half of the symmetric domain is shown. The conduction heater, shown in Figure 3 and used in experimental BLRO flow studies (Blunk, 1996), was constructed to create a temperature distribution on its surface that promotes $x$-directional BLRO flow. The surface temperatures vary in the $x$-direction and are uniform in the $y$-direction, reducing the situation to a 2-D problem. Temperatures vary spatially in the $x$-direction because of the air gap positioned below the glass plate in the centerline region. The rate of heat conduction from the electrical heater to the glass surface is reduced due to air's low thermal conductivity, resulting in lower glass temperatures in the centerline region.

The BLRO flow problem involves coupled momentum, heat, and mass transfer. B ecause surface tension depends on temperature and solvent concentration, heat and solvent conduction and convection to the free surface can affect the surface-tension-gradient driving-force. This convective transport in turn depends on the momentum diffusivity, which is also temperature and concentration dependent. Consequently, to obtain reproducible experimental results, the flow studies were conducted in an environmental chamber, in which air was forced over the heater surface at a sufficient velocity of $26 \mathrm{~cm} / \mathrm{s}$ in order to maintain the air temperature at ambient conditions and the air solvent concentration at zero. The airflow geometry, together with these constant air conditions at the free surface, are similar to those used in boundary-layer theory, enabling the heat- $\left(h_{1}\right)$ and mass-transfer $(k)$ coefficients to be found from theoretical correlations (Schlichting, 1979). A natural-convection correlation is used to estimate the heat-transfer coefficient $\left(h_{2}\right)$ for heat loss from the heater side (H olman, 1986).

\section{Solution Methodology}

The transient, incompressible, 2-D BLRO flow is governed by the continuity, momentum, energy, and mass equations, along with the boundary and initial conditions. Numerical solutions use both the finite-element (FEM) and the finite-difference approximation (FDA) methods. In the 1-D and 2-D codes, heat transfer within the conduction heater is approximated using the FEM ; momentum, energy, and mass transfer within the liquid film are approximated using the FDA. N ote that the full 2-D energy and mass-transfer equations had to be solved in the 1-D code in order to determine the temperature- and concentration-induced driving forces. The temperatures on the surface of the conduction heater, predicted from the FEM analysis, are used as essential input boundary conditions for the FDA analysis. A cubic spline-fit of the FEM temperatures is done to facilitate remeshing of the FDA domain. Note that an existing, general-purpose FEM code was modified and used to model heat transfer within the conduction heater (Wilkes, 1996).

The primitive variable (pressure/velocity) approach, as opposed to the stream function/vorticity $(\psi / \omega)$ approach, is used to formulate the governing momentum equations, although the latter approach is used to validate the 2-D code on a cavity-driven-flow test problem (Blunk, 1996). The primitive variable approach directly gives the desired pressure distribution within the film, and also facilitates incorporation of the free-surface boundary condition.

\section{BLRO Flow Assumptions}

The following assumptions, discussed fully by Blunk (1996), are used in the analysis:

- The polymer solutions (film material) are ideal. Enthalpy and volume changes on mixing are negligible.

- The flow is inertia-free. The Reynolds number is on the order of $10^{-6}$, based on the small film thicknesses used and the small film velocities ( $\approx 10^{-3} \mathrm{~cm} / \mathrm{s}$ ) observed experimentally using fluorescence microscopy.

- Evaporative cooling effects are negligible. Low-volatility solvents and low surface temperatures are used in this work.

- The shear stresses on the film surface, due to the flowing air stream, are insignificant compared to the surfacetension-gradient stresses.

- The film materials are Newtonian. The film viscosities are shear-rate independent up to $500 \mathrm{~s}^{-1}$ and shear rates of less than $1 \mathrm{~s}^{-1}$ are typical for leveling and sagging flows (Bauer, 1984).

- The solvent concentration at the film surface is at thermodynamic equilibrium and is not kinetically-controlled, that is, solvent diffusion is not rate-limiting.

- The binary mass diffusivity is independent of temperature and concentration.

- The thermal diffusivity is independent of temperature in the temperature range of $20^{\circ} \mathrm{C}$ to $90^{\circ} \mathrm{C}$ used.

- The glass surface temperatures are constant, within a small time step, and are not influenced by heat convection and diffusion in the film. The rate of thermal energy transferred into the thick glass substrate from the electrical heaters is significantly greater than that transported by the thin, creeping film. 


\section{Free-Surface Problem}

A review of numerical methods for solving viscous flows with moving boundaries can be found elsewhere (Floryan and R asmussen, 1989). Coordinate mapping, as opposed to the marker-and-cell (M A C) technique (H arlow and W elch, 1965) and the adaptive grid method (K istler and Scriven, 1984), is used to track the free surface, which enables the unknown irregularly-shaped, physical flow domain to be transformed onto a fixed regularly-shaped, computational domain. $M$ apping complicates the discretized form of the governing differential equations and boundary equations, because the unknown mapping function appears explicitly in these equations, but simplifies matters in tracking the free surface and in eliminating interpolation inaccuracies associated with a nonuniform grid. Since the $x$-directional grid-point locations do not change with time for the BLRO problem, a simple 1-D mapping function is required, which simply compresses or stretches the mesh in the z-direction. The transformation is

$$
x=x^{*}, \quad z=h(x, t) z^{*}
$$

where $h(x, t)$ represents the mapping function locating the free surface or the film thickness. The domain then transforms from

$$
0<\mathrm{x}<\mathrm{L}, \quad 0<\mathrm{z}<\mathrm{h}(\mathrm{x}, \mathrm{t})
$$

to

$$
0<\mathrm{x}^{*}<\mathrm{L}, \quad 0<\mathrm{z}^{*}<1,
$$

where $L$ is the maximum length in the $x$-direction. The free surface is always at $z^{*}=1$ when working in the computational domain. The time and spatial derivatives in the computational domain, which are required in the governing equations and boundary conditions, can be found elsewhere (Blunk, 1996). The Laplacian operator in the computational domain contains mixed derivatives that lead to numerical inefficiencies because the efficient, implicit alternating-direction method of Douglas-R achford (1956), together with the Thomas or tridiagonal-matrix algorithm (TDMA), can no longer be used to generate and solve, respectively, the resulting system of linear equations. Instead, the less-efficient Gauss-Seidel iterative method is used.

\section{Numerical Code Developments Governing equations (2-D code)}

The 2-D continuity, momentum, energy, and mass equations in the physical domain are

$$
\begin{gathered}
\frac{\partial \mathrm{u}}{\partial \mathrm{x}}+\frac{\partial \mathrm{w}}{\partial \mathrm{z}}=0 \\
\frac{\partial \mathrm{u}}{\partial \mathrm{t}}=-\frac{1}{\rho} \frac{\partial \mathrm{p}}{\partial \mathrm{x}}+\nu\left(\frac{\partial^{2} \mathrm{u}}{\partial \mathrm{x}^{2}}+\frac{\partial^{2} \mathrm{u}}{\partial \mathrm{z}^{2}}\right) \\
\frac{\partial \mathrm{w}}{\partial \mathrm{t}}=-\frac{1}{\rho} \frac{\partial \mathrm{p}}{\partial \mathrm{z}}+\nu\left(\frac{\partial^{2} \mathrm{w}}{\partial \mathrm{x}^{2}}+\frac{\partial^{2} \mathrm{w}}{\partial \mathrm{z}^{2}}\right)-\mathrm{g}
\end{gathered}
$$

$$
\begin{gathered}
\frac{\partial \mathrm{T}}{\partial \mathrm{t}}+u \frac{\partial \mathrm{T}}{\partial \mathrm{x}}+w \frac{\partial \mathrm{T}}{\partial \mathrm{z}}=\alpha\left(\frac{\partial^{2} \mathrm{~T}}{\partial \mathrm{x}^{2}}+\frac{\partial^{2} \mathrm{~T}}{\partial \mathrm{z}^{2}}\right), \\
\frac{\partial \mathrm{C}}{\partial \mathrm{t}}+\mathrm{u} \frac{\partial \mathrm{C}}{\partial \mathrm{x}}+\mathrm{w} \frac{\partial \mathrm{C}}{\partial \mathrm{z}}=\mathrm{D}_{\mathrm{sr}}\left(\frac{\partial^{2} \mathrm{C}}{\partial \mathrm{x}^{2}}+\frac{\partial^{2} \mathrm{C}}{\partial \mathrm{z}^{2}}\right),
\end{gathered}
$$

where $u$ and $w$ are the $x$ - and z-velocity components in the rectangular coordinate system, $T$ is the temperature, $C$ is the solvent concentration, $\mathrm{p}$ is the pressure, $\rho$ is the density, $\mathrm{t}$ is the time, $\mathrm{g}$ is the acceleration due to gravity, and $\nu, \alpha, \mathrm{D}_{\mathrm{sr}}$ are the momentum, thermal, and binary mass diffusivities, respectively. The subscripts $s$ and $r$ refer to solvent and resin. Note that the convective terms are absent in Eqs. 8 and 9 for this creeping flow.

At the free surface $z=h(x, t)$, the following three conditions exist

$$
\begin{aligned}
& \frac{\partial \mathrm{h}}{\partial \mathrm{t}}=\frac{1}{\mathrm{~A}^{2}}\left(-\mathrm{u} \frac{\partial \mathrm{h}}{\partial \mathrm{x}}+\mathrm{w}\right), \\
& \tau_{\tan }=\frac{\mu}{\mathrm{A}^{2}}\left\{\left(\frac{\partial \mathrm{u}}{\partial \mathrm{z}}+\frac{\partial \mathrm{w}}{\partial \mathrm{x}}\right)\left[1-\left(\frac{\partial \mathrm{h}}{\partial \mathrm{x}}\right)^{2}\right]\right. \\
&\left.+2 \frac{\partial \mathrm{h}}{\partial \mathrm{x}}\left(\frac{\partial \mathrm{w}}{\partial \mathrm{z}}-\frac{\partial \mathrm{u}}{\partial \mathrm{x}}\right)\right\}=\frac{1}{\mathrm{~A}}\left(\frac{\partial \sigma}{\partial \mathrm{x}}+\frac{\partial \mathrm{h}}{\partial \mathrm{x}} \frac{\partial \sigma}{\partial \mathrm{z}}\right), \\
& \tau_{\mathrm{n}}=\mathrm{p}_{\mathrm{a}}-\frac{2 \mu}{\mathrm{A}^{2}}\left\{\frac{\partial \mathrm{u}}{\partial \mathrm{x}}\left(\frac{\partial \mathrm{h}}{\partial \mathrm{x}}\right)^{2}-\left(\frac{\partial \mathrm{u}}{\partial \mathrm{z}}+\frac{\partial \mathrm{w}}{\partial \mathrm{x}}\right) \frac{\partial \mathrm{h}}{\partial \mathrm{x}}+\frac{\partial \mathrm{w}}{\partial \mathrm{z}}\right\} \\
&=-\frac{\sigma}{\mathrm{A}^{3}} \frac{\partial^{2} \mathrm{~h}}{\partial \mathrm{x}^{2}},
\end{aligned}
$$

where $\mathrm{A}=\sqrt{1+(\partial \mathrm{h} / \partial \mathrm{x})^{2}}, \mu$ is the viscosity, $\sigma$ is the surface tension, and $p_{a}$ is the atmospheric pressure. The kinematic condition ( $\mathrm{Eq}$. 12) describes how the film thickness changes with time, taking into account the $x$-directional movement of the surface. In Eq. 13, the tangential stress is equated to the surface-tension gradient (L evich-A ris boundary condition) and represents the driving-force for BLRO flow. The normal-stress boundary condition (E q. 14) results from a pressure discontinuity across an interface due to surface tension and a finite surface curvature. In this work, the atmospheric pressure is set equal to zero, since pressures are relative (not absolute) and only pressure gradients, and not pressures, appear in the momentum equations. Also, the denominators $A$ in all three surface equations are set to one because the surface slopes were found experimentally to be much less than one (maximum slope $\approx \pm 0.03$ ).

$\mathrm{H}$ eat and solvent losses via convection from the free surface are given by

$$
\begin{aligned}
& q=h_{1}\left(T-T_{\text {air }}\right), \\
& j=k\left(C-C_{\text {air }}\right),
\end{aligned}
$$

where $q$ and $j$ are the heat and mass flux, respectively, and $\mathrm{h}_{1}$ and $\mathrm{k}$ are the corresponding heat- and mass-transfer coefficients. The heat-transfer coefficient, calculated from 
boundary-layer theory, is assumed to be constant over the temperature range of $20^{\circ} \mathrm{C}$ to $90^{\circ} \mathrm{C}$ used in this study. The mass-transfer coefficient, on the other hand, is highly temperature dependent and was measured experimentally (Blunk, 1996).

In addition to these surface conditions, the following boundary conditions on the film must be imposed

$$
\begin{array}{lllll}
\mathrm{x}=0: & \mathrm{u}=0, & \partial \mathrm{w} / \partial \mathrm{x}=0, & \mathrm{q}=0, & \mathrm{j}=0, \\
\mathrm{x}=\mathrm{L}: & \mathrm{u}=0, & \mathrm{w}=0, & T=T_{f i x}, & \mathrm{C}=\mathrm{C}_{\mathrm{fix}}, \\
\mathrm{z}=0: & \mathrm{u}=0, & \mathrm{w}=0, & T=T_{\mathrm{FEM}}, & \mathrm{j}=0 .
\end{array}
$$

In Eq. 17, the z-momentum, heat- and mass-transfer conditions result from symmetry about the centerline. In E q. 18, it is assumed that the film length $L$ is sufficiently large that the film edge is unaffected by the gradients concentrated in the centerline region, resulting in zero velocities. The temperatures at the film edge (essential boundary condition) are determined from the glass and air temperatures, the external heat-transfer coefficient $h_{1}$, and the thermal conductivity. A linear variation of temperature through the film thickness is assumed. The concentrations at the edge are handled similarly. In Eq. 19, the no-slip momentum and the no-penetration mass conditions are used. A gain, the temperature at the bottom of the film is set equal to that of the conduction heater surface, which is obtained from the FEM analysis.

The initial conditions are a uniform film thickness, zero velocities, temperatures equal to that of the ambient air, and a uniform $14 \%$ volume concentration of solvent.

\section{Numerical method (2-D code)}

The pressure-correction algorithm SIMPLER (Semi-IM plicit Pressure Linking Equation R evised) is used to solve the momentum equations for velocities (Patankar, 1980). In this algorithm a pressure equation, generated from the continuity equation, is used to link the momentum and continuity equations. The calculated pressures correct the velocities iteratively until they satisfy the continuity equation. This algorithm, introduced and typically used with the finite-volume method, is used instead with the finite-difference method and the Gauss-Seidel iterative method with successive overrelaxation (SOR). In addition, SIM PLER is used with coordinate mapping, a uniform staggered grid, and border cells. The border cells and staggered grid were first introduced by $\mathrm{Har}$ low and Welch (1965) with the MAC method. The border cells are used to incorporate more easily the boundary conditions. The staggered grid is used, in effect, to generate a finer grid and also used to eliminate "wavy" velocity and pressure problems that may develop during numerical iteration, by allowing the dependent variables $(u, w, T, C, P)$ to be calculated at different locations in the domain (Patankar, 1980).

\section{Governing equation (1-D code)}

$\mathrm{H}$ ere the R eynolds equation, describing the time evolution of film thickness $h(x, t)$ for a thin, horizontal film subjected to surface-tension gradients, is derived based on the lubrication approximation. An order-of-magnitude analysis of the $\mathrm{N}$ avier-Stokes equations, using a small thickness-to-length ra- tio ( $h / L \ll 1)$ of the film geometry and the Levich-A ris freesurface boundary condition, reduces the $x$-momentum equation to Eq. 1 and the z-momentum equation considerably where only a hydrostatic pressure variation with $z$ is found (Papanastasiou, 1989). The pressure in the film consists of a surface-tension term modified by a hydrostatic effect

$$
p=-\sigma \frac{\partial^{2} h}{\partial x^{2}}+\rho g(h-z)
$$

where the pressure outside the liquid film is set to zero and $\partial^{2} \mathrm{~h} / \partial \mathrm{x}^{2}$ represents the surface curvature for small slopes. Assuming incompressibility, differentiation of the pressure with respect to $x$ gives

$$
\frac{\partial \mathrm{p}}{\partial \mathrm{x}}=-\sigma \frac{\partial^{3} \mathrm{~h}}{\partial \mathrm{x}^{3}}-\frac{\partial \sigma}{\partial \mathrm{x}} \frac{\partial^{2} \mathrm{~h}}{\partial \mathrm{x}^{2}}+\rho \mathrm{g} \frac{\partial \mathrm{h}}{\partial \mathrm{x}} .
$$

The nonlinear, 4th-order partial differential R eynolds equation for the film thickness is realized by substituting $\mathrm{Eq} .21$ into Eq. 4 to give

$$
\begin{aligned}
& \frac{\partial \mathrm{h}}{\partial \mathrm{t}}=-\frac{\mathrm{h}^{3} \sigma}{3 \mu} \frac{\partial^{4} \mathrm{~h}}{\partial \mathrm{x}^{4}}-\left(\frac{\mathrm{h}^{2} \sigma}{\mu} \frac{\partial \mathrm{h}}{\partial \mathrm{x}}+\frac{2 \mathrm{~h}^{3}}{3 \mu} \frac{\partial \sigma}{\partial \mathrm{x}}\right) \frac{\partial^{3} \mathrm{~h}}{\partial \mathrm{x}^{3}} \\
&-\left(\frac{\mathrm{h}^{2}}{\mu} \frac{\partial \sigma}{\partial \mathrm{x}} \frac{\partial \mathrm{h}}{\partial \mathrm{x}}+\frac{\mathrm{h}^{3}}{3 \mu} \frac{\partial^{2} \sigma}{\partial \mathrm{x}^{2}}-\frac{\mathrm{h}^{3} \rho \mathrm{g}}{3 \mu}\right) \frac{\partial^{2} \mathrm{~h}}{\partial \mathrm{x}^{2}} \\
&-\left(\frac{\mathrm{h}}{\mu} \frac{\partial \sigma}{\partial \mathrm{x}}-\frac{\rho \mathrm{gh}^{2}}{\mu} \frac{\partial \mathrm{h}}{\partial \mathrm{x}}\right) \frac{\partial \mathrm{h}}{\partial \mathrm{x}}-\frac{\mathrm{h}^{2}}{2 \mu} \frac{\partial^{2} \sigma}{\partial \mathrm{x}^{2}} .
\end{aligned}
$$

The initial and boundary conditions required to solve for $\mathrm{h}(\mathrm{x}, \mathrm{t})$ are

$$
\begin{aligned}
h(x, 0) & =h_{0}, \quad \text { (initial condition) } \\
\frac{\partial h}{\partial x}(0, t) & =0 \\
h(L, t) & =h_{0} \\
\frac{\partial h}{\partial x}(L, t) & =0 \\
\frac{\partial^{2} h}{\partial x^{2}}(L, t) & =0
\end{aligned}
$$

In Eq. 24, the thickness profile is symmetrical about the centerline. Equations 25 through 27 assume the profile is not affected by the surface-tension gradients at a large distance $L$ away from the centerline. However, after each time step, the film thickness for all $x$, including that in E q. 25, is adjusted to account for the solvent evaporated.

\section{Numerical method (1-D code)}

A numerical code based on the lubrication approximation was also developed. The lubrication approximation works well for thin-film flows and tapered-channel flows where there is 
a dominant velocity component in one direction. These two flows are similar in that they are inertia-free, generate pressure variations only in the primary flow direction, and use the resulting pressure distribution in an advantageous manner. These two flows are dissimilar in that gravitational effects are important typically in thin-film flows and not in taperedchannel flows.

The R eynolds equation ( $\mathrm{Eq}$. 22) is solved using:

(1) A finite-difference discretization method with 4th-order accurate approximations for the derivatives (Blunk, 1996).

(2) A variable grid size with a nonuniform grid in the $x$ direction to obtain accuracy in the high-gradient centerline region.

(3) The Newton-R aphson iteration method to linearize the discretized equations.

(4) The Gauss-Seidel iteration method with successive overrelaxation (SOR) to solve the set of linear equations.

When the film thickness profile is known, the $x$-velocity and pressure distributions are calculated using E qs. 1 and 20, respectively. The z-velocities are obtained from the continuity equation with the help of coordinate mapping. The full 2-D energy- and mass-transfer equations are then solved in the regular computational domain to determine the new surface-tension-gradient driving-force, which depends highly on temperature and solvent transfer to the free surface. Note that the Reynolds equation can be solved and the thickness profile can be adjusted for solvent loss only if the surface tension, the viscosity, and the solvent evaporation rate are known as functions of temperature and concentration.

\section{Materials}

Surface tensions and viscosities were measured with a D uN ouy Ring Tensiometer (Cenco No. 70535) and a Haake M 10 cup-and-bob viscometer, respectively, on solvent-free and solvent-filled coating materials. E vaporation studies were conducted with the environmental chamber under identical conditions used in the flow studies in order to measure the external mass-transfer coefficient accurately. Three polybutylene resins (Cannon N 1000, S2000, N 4000) and four solvents (1-methylnaphthalene, decane, undecane, tridecane) were used in the coating formulations to demonstrate the effects of viscosity, solvent volatility, initial film thickness, solventto-resin surface tension ratio, and heating rate on BLRO (Blunk, 1996). R esults indicate that the coatings were N ewtonian and the evaporation mechanism was surface-controlled, not diffusion-controlled (Blunk, 1996). H ence, the latter suggests that the value of the external mass-transfer coefficient is more critical than that of the diffusion coefficient. The diffusion coefficient was thus assumed to be temperature- and concentration-independent and set to a liquid-like value of $10^{-6} \mathrm{~cm}^{2} / \mathrm{s}$.

Note that the cross-linking reaction is not studied here, even though it has an extremely large effect on BLRO. Cross-linking affects coating viscosity directly through the formation of a 3-D structure, and affects surface tension indirectly through a decrease in solvent diffusion to the surface. Nevertheless, the BLRO mechanism can be investigated effectively and the numerical code can be developed effectively without complicating matters by including curing effects, which can be incorporated in future work.

\section{Results and Discussion Numerical code comparison (1-D $v$ s. 2-D)}

Here, BLRO-thickness results and computation times for the 1-D lubrication code are compared to those for the 2-D pressure-correction code. The intent is to determine which code is to be used in the numerical-vs.-experimental study and in future paint screening and paint-development studies. A $n$ objective is to develop an efficient and practical numerical code that predicts BLRO accurately.

Code comparisons were performed using three test cases. The first two cases involve a solvent-free film under highBLRO flow conditions - thick film, low viscosity, large surface-tension gradients - to determine whether the 1-D code can predict large degrees of BLRO accurately. Heat-transfer effects within the conduction heater and the film layer are included in the second case and not in the first. The third case involves a solvent-filled film and rapidly changing (magnitude and direction) surface-tension gradients to see if the 1-D code can handle concentration effects effectively. Both codes used a uniform grid with 50 and 10 grid points in the $x$ and z-directions, respectively. The 1-D code used a nonuniform grid with a high grid density in the larger gradient (centerline) area, whereas the 2-D code used a uniform grid. Computations were performed on the Silicon Graphics workstation for the 1-D code and on a supercomputer (Cray Y -M P M 98) for the 2-D code.

\section{Case 1}

A solvent-free film was subjected to a time-independent shear-stress distribution, generated from a constant temperature profile. This profile, measured with an infrared radiometer at the end of a high heating rate ( $86 \mathrm{~W}$ ) experiment, produced the largest surface-tension gradients used here. The initial film thickness was $200 \mu \mathrm{m}$ and the film viscosity and density were set at $8 \mathrm{P}$ and $0.8 \mathrm{~g} / \mathrm{cm}^{3}$, respectively. Figure 4 shows the transient, film-thickness profiles, which were truncated from $6.35 \mathrm{~cm}$ to $3 \mathrm{~cm}$ along the $x$-axis for clarity. The thickness remains constant at $200 \mu \mathrm{m}$ for $\mathrm{x}$-values exceeding $3 \mathrm{~cm}$. Excellent agreement is observed between the two codes. Small thickness differences are observed, and increase with increasing time, as the 1-D code predicts a slightly lesser degree of BLRO (that is, smaller centerline-to-valley thickness) than does the 2-D code, suggesting that eliminating $x$ - and z-momentum terms in the lubrication code does not affect the thickness results significantly. N ote that both codes generate profiles that conserve area. The areas above (BLRO hump) and below (valley) the 200 micron-line, calculated by integrating the profiles numerically via Simpson's rule, are nearly equivalent (within $2 \%$ ).

\section{Case 2}

This case involves the materials and conditions used in an actual BLRO flow experiment (N 1000 polybutylene resin, 86 W power setting, $203 \mu \mathrm{m}$ initial thickness), which generated the greatest degree of BLRO observed in the study (Blunk, 1996). U nlike Case 1, the film was subjected to time-varying shear stresses as the film was heated from ambient conditions. The energy equation was solved to account for heat transfer within the conduction heater and the film layer. 


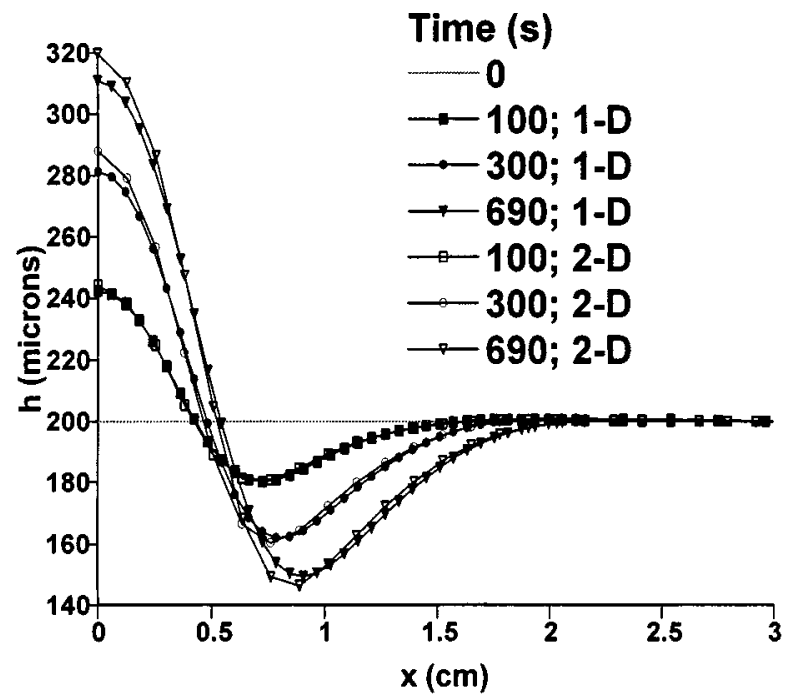

Figure 4. Case 1: film thickness profiles (1-D vs. 2-D) for a solvent-free film subjected to a timeindependent shear-stress distribution with a constant film viscosity and density of $8 \mathrm{P}$ and $0.8 \mathrm{~g} / \mathrm{cm}^{3}$, respectively.

1-D - filled symbols, 2-D - open symbols.

Profile results are presented in Figure 5. A gain, excellent agreement is observed between the 1-D and 2-D profiles, with the 1-D code predicting a slightly lower amount of BLRO than the 2-D code. The 2-D profile at $100 \mathrm{~s}$ is not apparent in the figure as this profile nearly coincides with the 1-D profile because of the scaling used to present the data. As in Case 1, the 1-D and 2-D profiles conserve area to within $2 \%$.

\section{Case 3}

Here, the materials and conditions were similar to those used in a BLRO flow experiment except that the mass-trans-

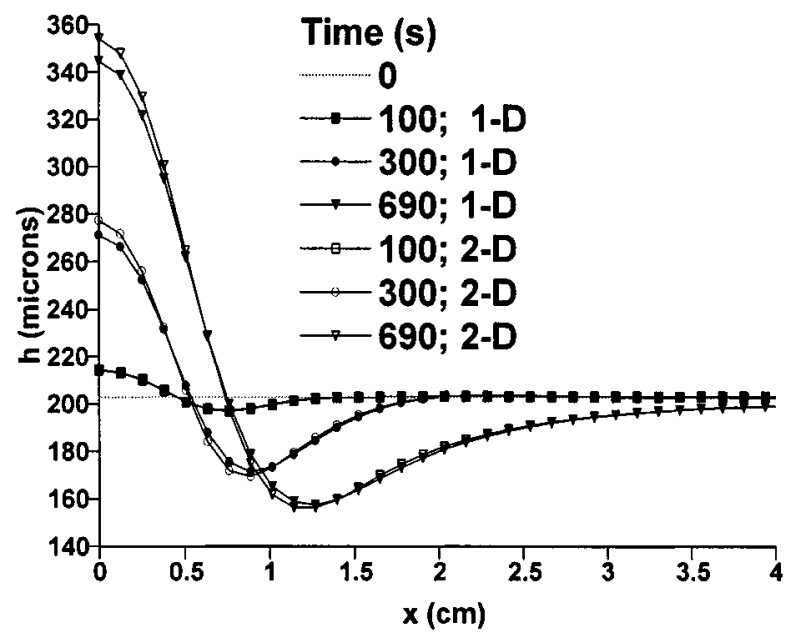

Figure 5. Case 2: film thickness profiles (1-D vs. 2-D) for a solvent-free film (N1000 polybutylene resin) subjected to large time-varying shear stresses.

1-D - filled symbols, 2-D - open symbols.

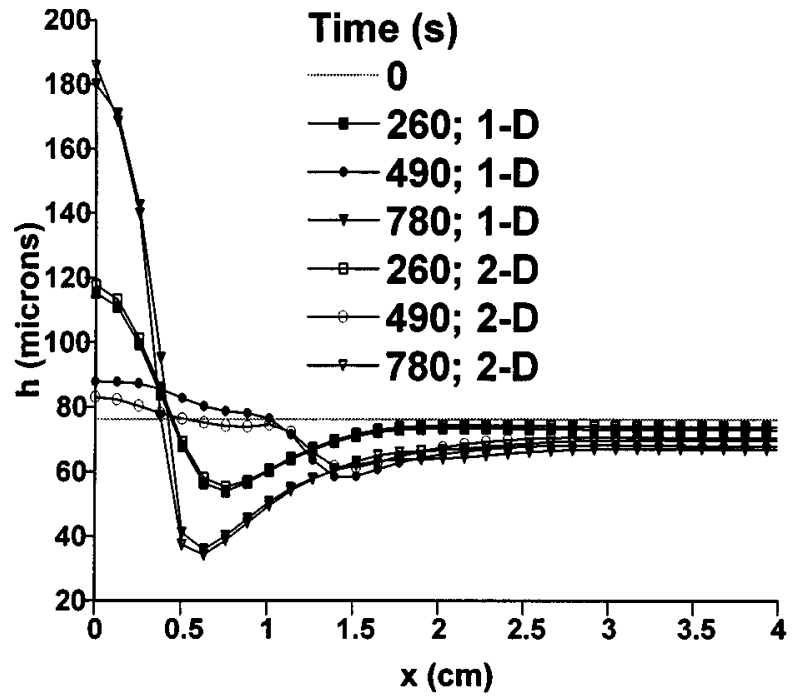

Figure 6. Case 3: film thickness profiles (1-D vs. 2-D) for a solvent-filled film (N1000 polybutylene resin dissolved in $14 \%$ by volume of undecane solvent) subjected to time-varying, temperature- and concentration-induced surface-tension-gradient forces.

1-D - filled symbols, 2-D - open symbols.

fer coefficient was increased by $20 \%$ to accentuate the effects of solvent concentration on BLRO flow developments. The film consists of $N 1000$ polybutylene resin dissolved in $14 \%$ by volume of undecane solvent. The conduction heater was heated at $86 \mathrm{~W}$ and an initial film thickness of $76.2 \mu \mathrm{m}$ was used. The film was subjected to time-varying, temperatureand concentration-induced surface-tension-gradient forces.

The film thickness profile results at three times are shown in Figure 6. Good agreement between the 1-D and 2-D profiles is observed. The three times correspond to three different modes of BLRO flow-formation at $260 \mathrm{~s}$, flow-out (leveling) at $490 \mathrm{~s}$, and reformation at $780 \mathrm{~s}$. During formation, the surface-tension gradients are temperature-induced, resulting in material accumulating in the cooler, higher surface-tension centerline area. During flow-out, the gradients are predominantly concentration-induced. Significant solvent concentration variations are generated along the film surface due to solvent evaporation from the nonuniform film thickness. The solvent concentration is greater in the thicker centerline area. Since the solvent surface tension is less than that of the resin, material flows from the centerline area towards the thinner, higher surface-tension areas. During reformation, the concentration variations diminish and once again, the surface-tension gradients are temperature-induced, resulting in material flow towards the cooler centerline area.

\section{Computing times}

The 1-D code is enormously more time-efficient than the 2-D code. In Case 3, where the fluid flow, energy, and masstransport equations are solved, $5.4 \mathrm{~min}$ (workstation) and 17 days (supercomputer) of CPU time are required for the 1-D 
and 2-D codes, respectively. This huge time difference results from a severe, time-step stability limitation with the 2-D code. Time steps of $1 \mathrm{~s}$ and $10^{-4} \mathrm{~s}$ were used for the 1-D and 2-D codes, respectively. Time steps exceeding $10 \mathrm{~s}$ for the 1-D code and $10^{-4} \mathrm{~s}$ for the 2-D code resulted in instabilities. In the 2-D code, extremely small time steps are required to analyze momentum transport within thin, viscous films according to von N eumann's stability criterion

$$
\Delta t<\frac{(\Delta z)^{2}}{\nu}
$$

where $\nu$ is the momentum diffusivity (kinematic viscosity) and $\Delta z$ is the grid spacing in the film-thickness direction (Carnahan et al., 1969). E quation 28 is based on a linear stability analysis, an explicit time scheme, and diffusion in one direction. If the time step is too large, the explicit procedure will be unstable. Even for analyses based on a fully implicit scheme, Eq. 28 provides a "rule-of-thumb" time-step estimate required to obtain accurate solutions, indicating that small time steps are required in 2-D codes to predict momentum transport accurately, regardless of whether an implicit or explicit time scheme is employed. N ote that since the thermal and mass diffusivities were four and six orders of magnitude lower than the momentum diffusivity, respectively, a time step as large as 1 to $10 \mathrm{~s}$ could be used in both codes to analyze heat and mass transfer without generating numerical instabilities and inaccuracies.

In the 2-D code, the "semi-implicit" pressure-correction algorithm SIM PLER was used. It is semi-implicit, however, in a nontraditional sense. A fully implicit scheme is used in time, because the unknown velocities at the current time were calculated indirectly or implicitly from neighboring unknown velocities, also at the current time, by solving a set of simultaneous equations. The method is considered semi-implicit because a velocity correction term was omitted in the derivation of the pressure-correction equation in order to adopt a sequential, one-variable-at-a-time, solution procedure instead of directly solving the entire set of momentum and continuity equations (Patankar, 1980). This semi-implicit method is susceptible to instabilities for large time steps. The authors recommend, however, that a fully implicit code (not a semi-implicit code-SIMPLER ) be used and its results compared to those calculated by the 1-D code to gain more insight into the efficiency of the lubrication-based, 1-D code.

In the remainder of this article the accurate and efficient 1-D code is used to provide the numerical BLRO results.

\section{Experimental vs. Numerical Results Film profiles}

A detailed description of the BLRO-flow experimental apparatus can be obtained elsewhere (Blunk, 1996). The major components, however, are: the conduction heater, which generates temperature gradients on its surface, and, in turn, surface-tension-gradients in the coating; the environmental chamber, which provides a controlled setting for heat and mass transfer; and the non-contact, laser line-scan profilometer (A nalytical M easurement Technology, M adison Heights, $\mathrm{MI}$ ), which measures the time-varying, wet-film surface pro-

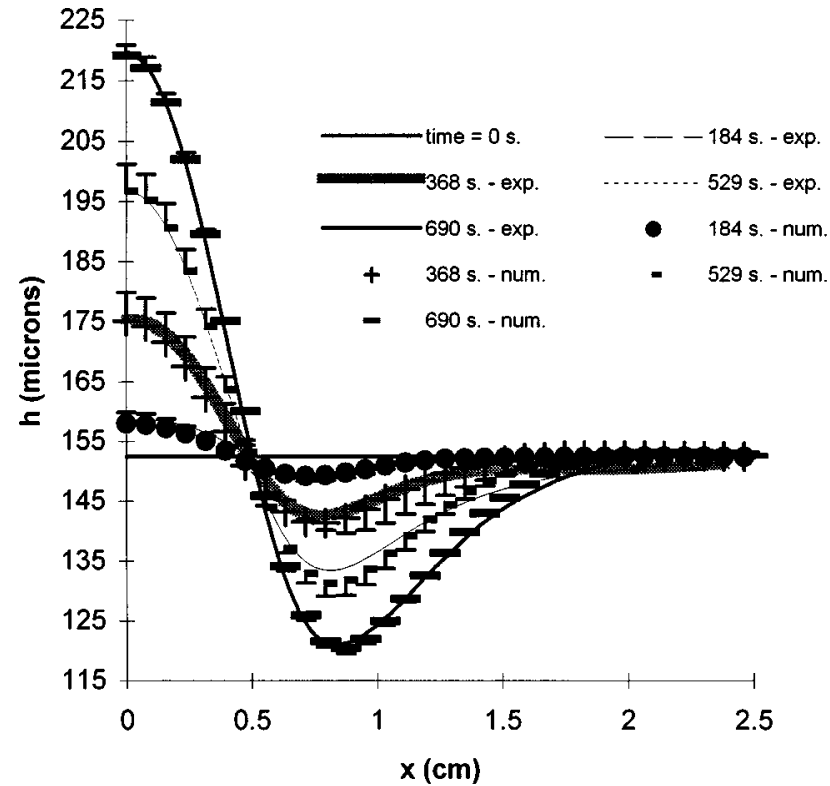

Figure 7. Experimental vs. numerical transient thickness profiles of a solvent-free film (S2000 polybutylene) heated at $58 \mathrm{~W}$.

O ne-sided error bars on numerical data to represent uncertainty in initial film thickness measurement.

files. Each line-scan profile contains 512 data points with 0.01 $\mathrm{cm}$ spacing.

Film-thickness profiles from two representative BLR O flow experiments-solvent-free and solvent-filled-are compared with those predicted numerically. The thickness values obtained from the solvent-filled experiment were reduced to account for the solvent evaporated, as the laser profilometer measures surface profiles, not coating thickness. The numerical code was used to provide the solvent loss information needed to make this reduction.

\section{Solvent-free experiment}

Figure 7 presents numerical and experimental profile results of an S2000 polybutylene film. The film with a measured initial thickness of $152 \mu \mathrm{m}$ was heated using a medium heating rate ( $58 \mathrm{~W}$ ). V ery good agreement is observed among the experimental and numerical profiles. O ne-sided error bars are displayed at each numerical datum point to illustrate the significance of a $25 \mu \mathrm{m}$ underestimation in measuring the initial film thickness. Here, the initial thickness was measured to be $152 \mu \mathrm{m}$ using a wet-film thickness gage ( $\mathrm{G}$ ardco, $M$ odel 154). The actual thickness could range anywhere from $152 \mu \mathrm{m}$ to $177 \mu \mathrm{m}$, because the probe lengths on the thickness gage were in $25 \mu \mathrm{m}$ increments. The numerical code was run twice using a $152 \mu \mathrm{m}$ and $177 \mu \mathrm{m}$ initial film thickness, and the resulting thicknesses from the latter analysis were then reduced by $25 \mu \mathrm{m}$ so that these measurementerror effects could be investigated. The small error bars shown in the figure suggest that the $25 \mu \mathrm{m}$ underestimation in initial thickness is insignificant under the conditions used in this experiment. In addition, the error bars suggest that the actual initial film thickness was probably closer to $152 \mu \mathrm{m}$ than to $177 \mu \mathrm{m}$, assuming no other experimental errors. 


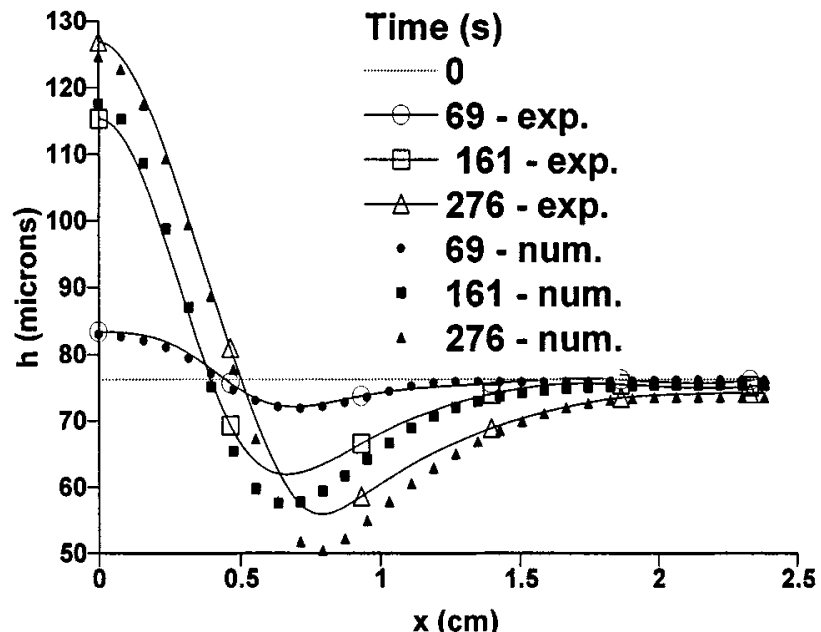

Figure 8. Experimental vs. numerical profile results of a solvent-filled film (N1000 polybutylene in $14 \%$ by volume undecane) during BLRO formation (86 $\mathrm{W}$ heating rate).

\section{Solvent-filled experiment}

A n N 1000 film, dissolved in $14 \%$ by volume undecane solvent, was heated at a high $86 \mathrm{~W}$ heating rate and had an initial film thickness between $76.2 \mu \mathrm{m}$ and $101.6 \mu \mathrm{m}$. A s expected, of all the conditions used in this work, these conditions generated the largest deviations between experimental and numerical work. High solvent volatility, high heating rate, and thin initial thickness all result in a large and rapid change in the surface-tension-gradient driving-force (magnitude and direction) as these conditions produced all three modes of BLRO flow. Small errors in the temperature- and concentration-dependent surface tension, viscosity, and solvent flux measurements, which are input for the numerical code, can affect the numerical results significantly and result in large experimental vs. numerical deviations. M oreover, a $25 \mu \mathrm{m}$ underestimation in the initial film thickness can also affect the resulting profiles significantly.

Figures 8, 9 and 10 present experimental and numerical profile results obtained during BLRO formation, flow-out, and reformation, respectively. A n initial thickness of $76.2 \mu \mathrm{m}$ was used in the numerical code. Good agreement is shown between the experimental and numerical results. Note that each experimental profile contains 512 data points. For clarity, six symbols are illustrated on each profile.

\section{Summary}

Based on the lubrication approximation, an efficient numerical 1-D code was developed to predict temperature- and concentration-induced, surface-tension-driven BLRO flows, which agreed with those predicted from a 2-D code and those measured from experiments. The lubrication approximation worked well because of the BLRO flow geometry, that is, small film thickness-to-length ratio $\mathrm{h} / \mathrm{L}$. The greatest asset of the 1-D code was that it was vastly more time-efficient and economical to run than running the 2-D code. Because of a severe time-step stability limitation associated with the 2-D

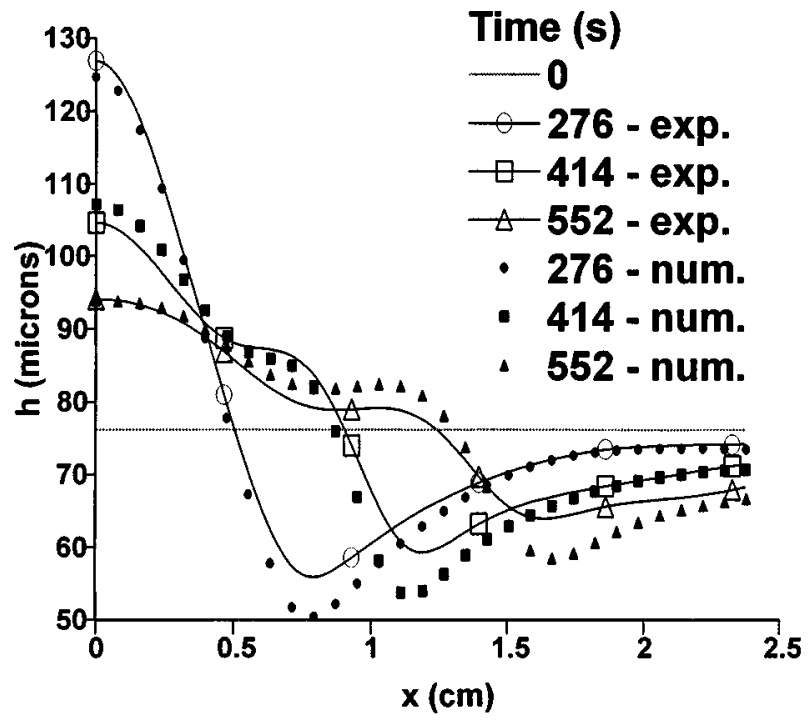

Figure 9. Experimental vs. numerical profile results of solvent-filled film (N1000 polybutylene in $14 \%$ by volume undecane) during BLRO flow-out (86 $\mathrm{W}$ heating rate).

code, and not with the 1-D code, the 1-D code simulated a 13-min BLRO flow experiment on a workstation in approximately four min, whereas the 2-D code required weeks on a supercomputer.

The 1-D code was written specifically to handle a simple coating system, namely, a nonreacting, single-solvent, singleresin liquid. The lubrication approximation in the 1-D code is also expected to handle more complex, automotive coating systems because these systems are found to produce BLRO of a lesser degree due to the rapid increase in viscosity with extent of cure. In order to model reacting, multiple-solvent

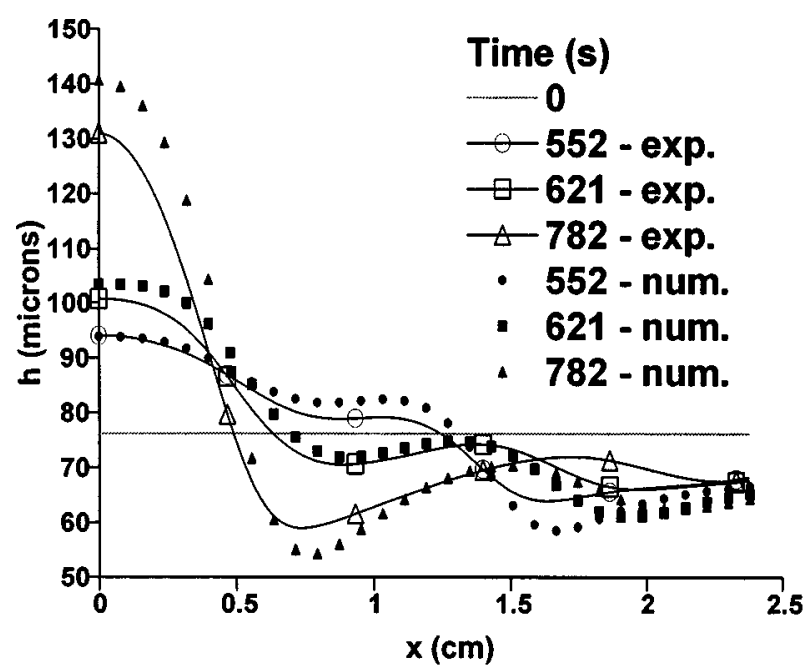

Figure 10. Experimental vs. numerical profile results of solvent-filled film (N1000 polybutylene in 14\% by volume undecane) during BLRO reformation (86 W heating rate). 
and multiple-resin automotive coatings accurately, effects of the following (in the order of increasing complexity) must be incorporated into the code:

(1) Y ield stress and other non-N ewtonian behavior; (2) viscosity and mass diffusivity dependency on cross linking; (3) for diffusion-controlled evaporation, the dependency of the mass diffusivity on solvent concentration and temperature; (4) multiple solvent diffusion; and (5) surfactant and low-energy substance (byproduct of curing reaction) diffusion.

\section{Literature Cited}

Bauer, D. R., "M echanism for Flow Control by Dispersed Polymeric Particles in High Solids Paints," Organic Coatings, 7, 157 (1984).

Bauer, D. R ., and L. M . B riggs, "R heological M odels for Predicting Flow in High Solids Coatings," J. Coatings Technol., 56, 87 (1984).

Biermann, V. M., "D as Fliessen Ebener Schichten Inkompressibler $\mathrm{V}$ iscoelasticher Flussigkeiten mit einer in A blaufrichtung Infinitesimal G estorten Freien Oberflache," Rheol. Acta, 7, 138 (1968).

Blunk, R. H. J., "Surface-Tension-D riven Flows of Coatings: Bondline R eadout Formation," PhD Diss., U niv. of Michigan, A nn A rbor, MI (1996).

Carnahan, B., H. A. Luther, and J. O. Wilkes, Applied Numerical M ethods, Wiley, N ew Y ork (1969).

D egani, D., and C. G utfinger, "A N umerical Solution to the Leveling Problem," Comput. Fluids, 4, 149 (1976).

D ouglas, J., J r., and H. R achford, J r., "On the N umerical Solution of Heat Conduction Problems in Two and Three Space Variables," Trans. Amer. M ath. Soc., 82, 421 (1956).

Eley, R. R., D. E W eidner, and L. W. Schwartz, "R ole of Surface Tension Gradients in Correcting Coating Defects in Corners," J. Colloid and Interface Sci., 179, N 1 (1996).

Ellwood, K. R., "The M echanics and Stability of Liquid J ets and Films," PhD Diss., U niv. of Michigan, A nn A rbor, MI (1991).
Fink-J ensen, P., "Surface Disturbances in Fluid Paint Films," Farg och Lack, 8, 5 (1962).

Floryan, J. M., and H. R asmussen, "Numerical M ethods for $V$ iscous Flows with M oving Boundaries," Appl. Mech. Rev., 42, 323 (1989).

$\mathrm{H}$ arlow, F. H., and J. E. W elch, "N umerical Calculation of Time-D ependent V iscous Incompressible Flow of Fluid with Free Surface," Phys. of Fluids, 8, 2182 (1965).

H olman, J. P., H eat Transfer, 6th ed., M cG raw-H ill, N ew Y ork (1986).

J Oos, F. M., "Leveling of a Film with Stratified V iscosity and Insoluble Surfactant," AIChE J., 42, 623 (1996).

K heshgi, H. S., and L. E. Scriven, "Penalty Finite-E lement A nalysis of U nsteady Free Surface Flows," Finite Elements in Fluids, J. T. O den, ed., Wiley, N ew Y ork (1983).

Kistler, S. F., and L. E. Scriven, "Coating Flow Theory by Finite Element and A symptotic A nalysis of the Navier-Stokes System," Int. J. Numer. Methods Fluids, 4, 207 (1984).

Keunings, R., and D. W. Bousfield, "A nalysis of Surface-TensionDriven Leveling in V iscoelastic Films," J. Non-N ewt. Fluid Mech., 22, 219 (1987).

M arangoni, C. G. M., Ann. Phys. (Poggendorff), 143, 337 (1872).

Orchard, S. E., "On Surface Levelling in V iscous Liquids and Gels," J. A ppl. Sci. Res., A, 11, 451 (1962).

O verdiep, W. S., "The Leveling of Paints," Prog. in Org. Coatings, 14, 159 (1986).

Papanastasiou, T. C., "Lubrication Flows," ChE Ed., 50 (1989).

Patankar, S. V ., N umerical H eat Transfer and Fluid Flow, H emisphere Publishing Company (1980).

Pierce, P. E., and C. K. Schoff, "Coating Film Defects," Federation Series on Coatings Technology, 7 (1988),

Schlichting, H., Boundary Layer Theory, 7th ed., M cG raw-Hill, New Y ork (1979).

Wilkes, J. O., Applied Numerical Methods, U niv. of M ichigan ChE 508 Notes, U niv. of M ichigan, A nn A rbor, M I, 7 (1996).

M anuscript received Mar. 13, 2000, and revision received Oct. 18, 2000. 